\title{
Branchial Cleft Cyst Carcinoma: A Case Report
}

\author{
Hyunji Kim, ${ }^{1}$ Hyun Sook Hong, ${ }^{1,}{ }^{*}$ Jeong Ja Kwak, ${ }^{2}$ Seung Won Lee, ${ }^{3}$ and Sun Hye Jeong ${ }^{1}$ \\ ${ }^{1}$ Department of Radiology, Soonchunhyang University Bucheon Hospital, Bucheon, South Korea \\ ${ }^{2}$ Department of Pathology, Soonchunhyang University Bucheon Hospital, Bucheon, South Korea \\ ${ }^{3}$ Department of Otolaryngology/Head and Neck Surgery, Soonchunhyang University Bucheon Hospital, Bucheon, South Korea \\ "Corresponding author: Hyun Sook Hong, Department of Radiology, Soonchunhyang University Bucheon Hospital, Soonchunhyang University College of Medicine, Bucheon, \\ Korea. Tel: +82-326215851, Fax: +82-326215874, E-mail: hshong@schmc.ac.kr
}

Received 2016 June 25; Accepted 2016 July 26.

\begin{abstract}
Branchial cleft cyst carcinoma (BCCC) is an extremely rare malignancy originating from cells within the branchial cleft cyst wall. A 73-year-old man presented with a cystic mass with cellulitis mimicking abscess initially and recurred 3 years later as complex cystic lesion in right neck level II with multiple necrotic ipsilateral lymphadenopathy. The pathological diagnosis of cystic lesion was squamous cell carcinoma, suggesting possibility of originating from branchial cleft cyst. There was no identifiable primary cancer elsewhere by a thorough evaluation; eventually final diagnosis was branchial cleft cyst carcinoma to meet the modified criteria of Khafif et al. Up to the present time, there has been no evidence of recurrence. Although the BCCC is very rare, accurate diagnosis is important to plan proper treatment for patient. This report should help increase awareness of BCCC, which should be included in the differential diagnosis of a cystic neck mass.
\end{abstract}

Keywords: Head and Neck Neoplasms, Diagnosis Differential Branchioma, Carcinoma, Squamous Cell, Lymphatic Metastasis

\section{Introduction}

Branchial cleft cyst carcinoma (BCCC) is a rare malignancy originating from cells within the cyst, located in anterior aspect of sternocleidomastoid muscle, posterior to submandibular gland and lateral to carotid sheath. Initially described by Volkmann in 1882, it was better defined by Martin et al. (1) in 1950, and the definition was refined by Khafif et al. (2). Nevertheless, confusion about this entity continues, because 1 ) the incidence of metastatic squamous cell carcinoma is far greater than that of branchial cleft carcinomas, 2) most branchial cysts and jugular lymphatics are presented in similar areas, 3) metastatic lymphadenopathy may be the first finding of an occult head and neck cancer, and 4) BCCC and necrotic lymph node can be difficult to differentiate on histology.

Neck masses can be classified as inflammatory, congenital, and neoplastic according to patient's age, anatomical location, duration of the neck mass, and the associated clinical findings. In patients older than 40 years presenting with a neck lump, the mass is neoplastic in roughly $80 \%$ of all non-thyroid neck masses, and of these neoplastic lesions, approximately $80 \%$ are malignant (3). This report should help radiologic differential diagnosis of a cystic neck mass.

\section{Case Presentation}

A 73-year-old man complained of a swelling with pain in the right upper neck for 10 days. Physical examination showed tenderness with local warmth. He had a gingival injury caused by his dentures before developing the lesion. The laboratory findings included a white blood cell count of $14,100 / \mu \mathrm{L}$ with $81.2 \%$ neutrophils. Contrast-enhanced computed tomography (CT) showed a marginally enhancing low-attenuated mass in the anterior border of the sternocleidomastoid muscle at the level of hyoid bone, approximately $3 \mathrm{~cm}$ in diameter, which was displacing the right submandibular gland anteriorly and carotid sheath medially (Figure 1A). The ipsilateral platysma muscle was thickened, and there was surrounding fat infiltration and ipsilateral lymphadenopathy. This was diagnosed as an abscess and incision, and drainage was performed. On followup CT, the size of the abscess cavity had decreased.

Three years later, the patient presented with a recurrent right neck mass in the same location. CT showed a 3 $\mathrm{cm}$ rim-enhancing low-density mass with enhancing solid septa in the same location in the right neck and necrotic lymph nodes at neck levels II and III, which were suggestive of metastatic lymphadenopathy (Figure 1B). A fineneedle aspiration biopsy was suggestive of an inflamed branchial cleft cyst. An excisional biopsy was performed to remove an approximately $4 \times 3 \mathrm{~cm}$ cystic mass. The frozen biopsy showed squamous cell carcinoma. Therefore, additional pathology specimens were obtained, including a triple endoscopic biopsy of the tonsillar fossa, tongue base, nasopharynx, and hypopharynx. There was no evidence of malignancy or metastasis at these other sites. No primary malignancy was found on contrast-enhanced CT or 

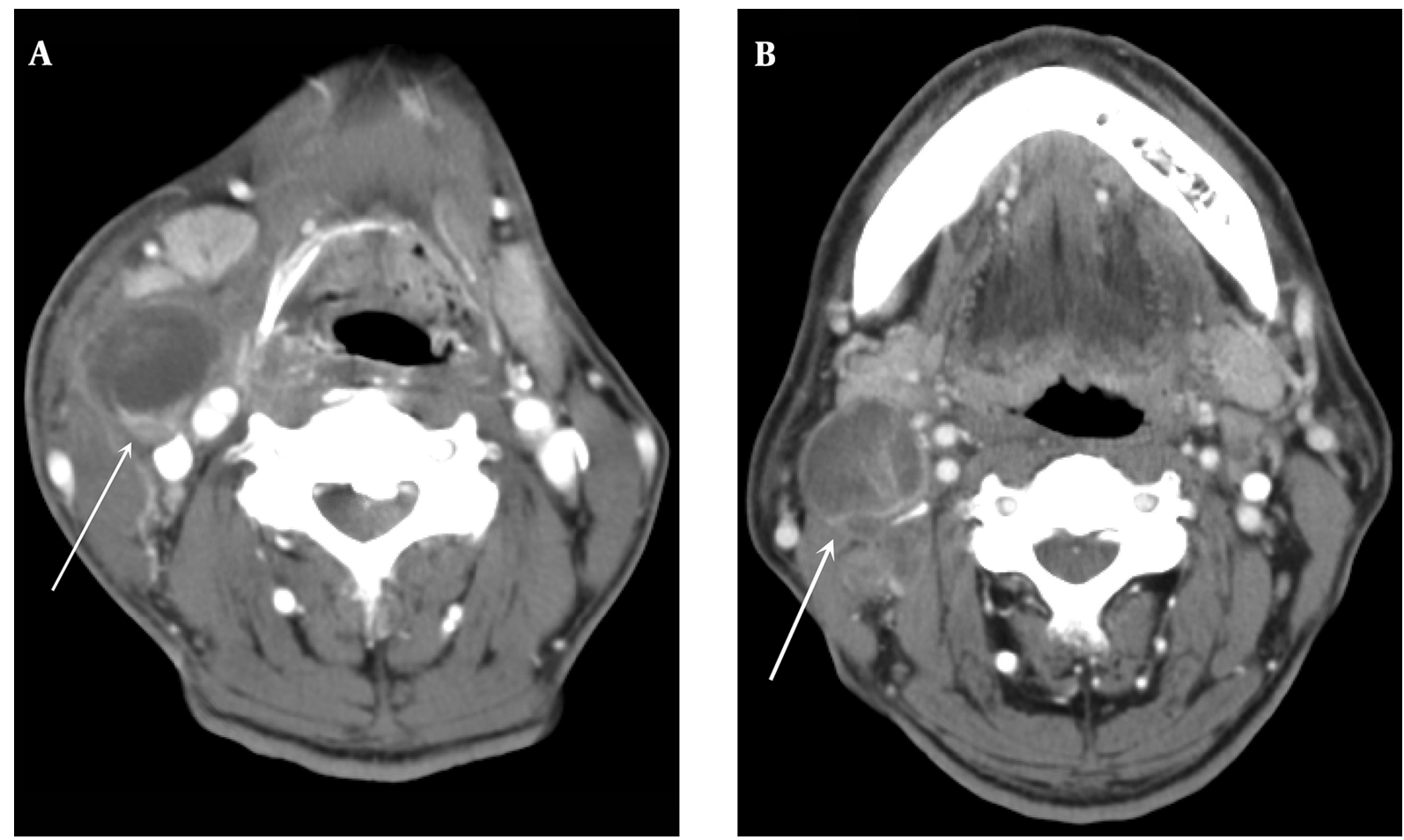

Figure 1. A 73-year-old man presented with a painful swelling in the right upper neck for 10 days. A, Contrast-enhanced CT showed a marginal enhancing cystic mass (arrow) in the anterior border of the sternocleidomastoid muscle that was displacing the right submandibular gland anteriorly and carotid sheath medially. Thickened ipsilateral platysma muscle and surrounding fat infiltration are seen. B, Three years later, he presented with a recurrent right neck mass in the same location. CT showed a $3 \mathrm{~cm}$ rimenhancing low-density mass with enhancing solid septa (arrow) and necrotic lymph nodes at neck levels II and III.

positron emission tomography CT. He subsequently underwent bilateral modified radical neck dissection, and multiple metastatic lymph nodes were identified in the left neck at levels III and IV.

The resected specimen was a $4.2 \times 2.8 \mathrm{~cm}$ cystic mass with a smooth gray surface. The cut surface had an irregular cystic structure with several gray nodules in the cyst wall. Microscopically, the cystic structure was lined by squamous epithelium and presented many hyperplastic lymphoid follicles with germinal centers in the cyst wall. The cyst epithelium revealed transition from benign normal epithelium to carcinoma in situ (Figure 2A and B).

Ultimately, this was diagnosed as branchial cleft cyst carcinoma. The patient refused postoperative adjuvant radiation therapy. At the time of this report, there has been no evidence of recurrence.

\section{Discussion}

Cystic lesions in the neck are encountered frequently in clinical practice, and raise diagnostic challenge. Majority of cystic lesions in young adults are congenital, however, about $80 \%$ of the cystic lesions in patients over
40 years of age are malignancy (4). Metastatic lymphadenopathy undergoing cystic change from squamous cell carcinoma from upper aerodigestive tract may mimic a branchial cleft cyst on imaging (5). The incidence of metastatic lymphadenopathy undergoing cystic degeneration from Waldeyer's ring is $33-62 \%(5,6)$. A single cystic metastasis in the upper lateral neck may disguise as BCCC if there is no apparent primary tumor on the initial evaluation. The reported incidence of unexpected carcinoma in cervical cysts, initially diagnosed as BCCC, is $4-24 \%$ (7).

Goyal et al. (3) evaluated the CT characteristics of BCCC and cystic lymph node metastasis. There was a significant difference in the age distribution of the two populations, with the BCCC population being much younger. Untypical features such as heterogeneity, inner septation, extracapsular spread, or a thick outer wall suggest malignancy of a cystic lesion (3). In our case, the recurrent cystic mass contained internal enhancing septa and had an asymmetrically thickened wall, and there were multiple necrotic lymph nodes in the neck. These findings differ from those of the initial cystic mass, and we retrospectively postulate that these implied malignant potential. Given this degree of overlap, a high level of suspicion is necessary when eval- 

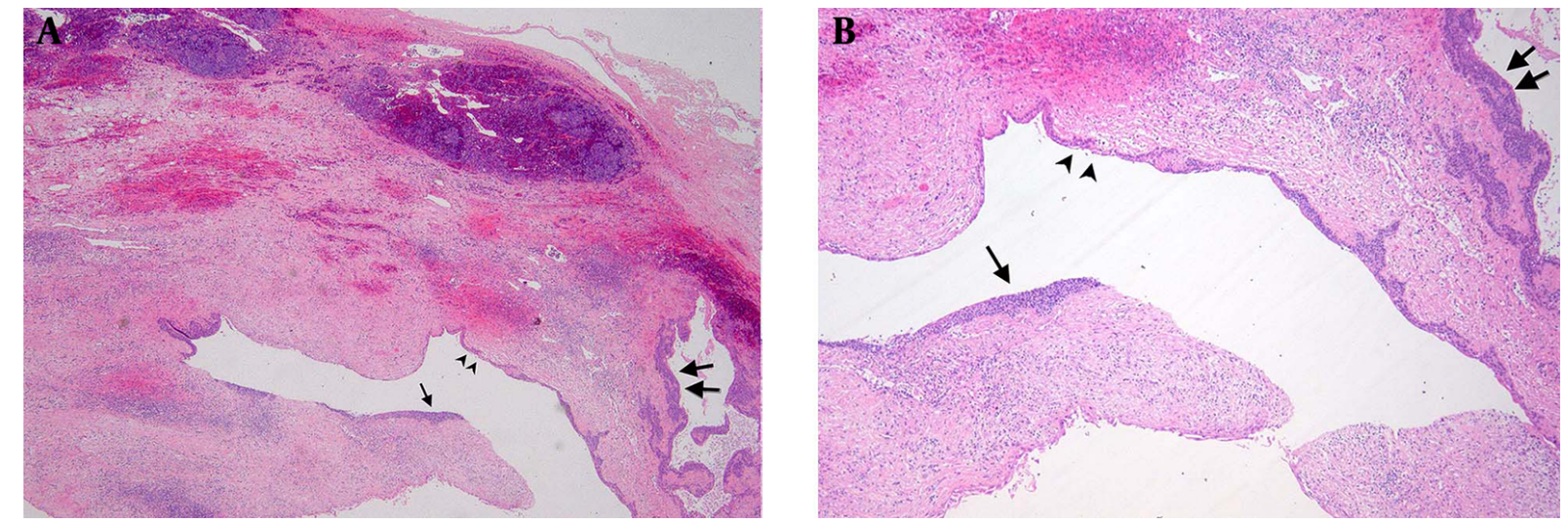

Figure 2. A and B, The cyst is lined by normal squamous epithelium (arrow head), dysplastic epithelium (arrow) and squamous cell carcinoma in situ (double arrows). The cystic wall shows fibrous tissue and activated lymphoid follicles (hematoxylin-eosin stain, $\times 40$ (A), $\times 100$ (B)).

uating cystic neck masses, especially in older individuals.

BCCC arises from the remnants of the branchial clefts or pharyngeal pouches. As the cyst is usually lined with stratified squamous epithelium, malignant transformation in the epithelium is feasible; this is known as BCCC and is considered extremely rare $(3,8)$.

Martin et al. (1) analyzed 250 cases reported in the English literature from 1882 until 1950 and proposed criteria for the diagnosis of BCCC. Based on a retrospective review of 67 additional cases reported from 1950 to 1988, Khafif et al. (2) added to the criteria of Martin et al. (1):

1. The tumor is located in the anatomic region of the branchial cleft cyst or sinus, as defined by Martin et al. (1).

2. The histological appearance of the tumor is consistent with its origin from branchial vestiges, i.e., squamous cell carcinoma.

3. Carcinoma is present within the lining of an identifiable epithelial cyst.

4. There is a transition from the normal squamous epithelium of the cyst to carcinoma.

5. No identifiable primary malignant tumor is found in an exhaustive evaluation; this must include endoscopy (nasopharyngoscopy, laryngoscopy, bronchoscopy, and esophagoscopy), radiographic examinations, full CT of the head and neck, and appropriate biopsies.

The crucial criterion emphasized by Khafif et al. (2) is the tumor histology: a cystic structure partially lined by normal squamous or sometimes pseudostratified columnar epithelium, with increasing atypia and dysplasia and a gradual transition through intraepithelial carcinoma to invasive squamous cell carcinoma with pleomorphism, multiple mitoses, and infiltration. According to the criteria of Khafif et al. (2) only 10 cases from 1951 to 1988 were identified true branchiogenic carcinomas. Thomp- son found that less than 25 cases have been proved as BCCC since 1988 according to Khafif's criteria (8). Our case satisfies the criteria by Khafif et al. (2) regarding the anatomical location, transition from benign normal epithelium to invasive squamous cell carcinoma, and absence of an primary tumor under scrutiny.

This patient presented with a cystic lesion mimicking an abscess, which recurred 3 years later as a cystic mass in the lateral neck with multiple necrotic lymph nodes. We could not determine when the cystic mass transformed into carcinoma, but multiple necrotic lymphadenopathy is a clue for the diagnosis of malignant transformation.

In conclusion, when we encounter a cystic neck mass in older individuals, we need to suspect malignancy and search for other ancillary findings.

\section{Footnotes}

Authors' Contributions: Study concept and design: Hyun Sook Hong, acquisition of data: Hyunji Kim, Jeong Ja Kwak, Seung Won Lee, Sun Hye Jeong, analysis and interpretation of data: Hyun Sook Hong, Jeong Ja Kwak, Seung Won Lee, Sun Hye Jeong, drafting of the manuscript: Hyunji Kim, Hyun Sook Hong, critical revision of the manuscript for important intellectual content: Hyun Sook Hong, study supervision: Hyun Sook Hong

Financial Disclosure: None declared.

Funding/Support: This work was supported in part by the Soonchunhyang University research fund.

\section{References}

1. Martin H, Morfit HM, Ehrlich H. The case for branchiogenic cancer (malignant branchioma). Ann Surg. 1950;132(5):867-87. [PubMed: 14771797]. 
2. Khafif RA, Prichep R, Minkowitz S. Primary branchiogenic carcinoma. Head Neck. 1989;11(2):153-63. [PubMed: 2656582].

3. Goyal N, Zacharia TT, Goldenberg D. Differentiation of branchial cleft cysts and malignant cystic adenopathy of pharyngeal origin. AJR Am J Roentgenol. 2012;199(2):W216-21. doi: 10.2214/AJR.11.8120. [PubMed: 22826424].

4. Granström G, Edström S. The relationship between cervical cysts and tonsillar carcinoma in adults. J Oral and Maxillofacial Surgery. 1989;47(1):16-20.

5. Flanagan PM, Roland NJ, Jones AS. Cervical node metastases presenting with features of branchial cysts. J Laryngol Otol. 1994;108(12):1068-
71. [PubMed: 7861083].

6. Goldenberg D, Sciubba J, Koch WM. Cystic metastasis from head and neck squamous cell cancer: a distinct disease variant? Head Neck. 2006;28(7):633-8. doi:10.1002/hed.20381. [PubMed: 16477605].

7. Gourin CG, Johnson JT. Incidence of unsuspected metastases in lateral cervical cysts. Laryngoscope. 2000;110(10 Pt 1):1637-41. doi: 10.1097/00005537-200010000-00012. [PubMed: 11037817].

8. Thompson LDR, Heffner DK. The Clinical Importance of Cystic Squamous Cell Carcinomas in the Neck. Cancer. 1998;82(5):944-56. [PubMed: 9486586]. 